\title{
Elementary Model of Constraint Quantization with an Anomaly
}

\author{
J. Scott Little* and John R. Klauder ${ }^{\dagger \ddagger}$ \\ Department of Physics \\ University of Florida \\ Gainesville, FL 32611
}

\begin{abstract}
Quantum gravity is made more difficult in part by its constraint structure. The constraints are classically first-class; however, upon quantization they become partially second-class. To study such behavior, we focus on a simple problem with finitely many degrees of freedom and demonstrate how the projection operator formalism for dealing with quantum constraints is well suited to this type of example.
\end{abstract}

\section{Introduction}

It is well known that the constraint algebra for classical gravity is first-class; however, following the standard Dirac prescription for the quantization of constraints, the resultant quantum constraint algebra is partially secondclass [1. Since the Dirac procedure is better adapted to quantum first-class systems, the partial second-class character causes difficulties when discussing this problem. Another procedure for constraint quantization is known as the projection operator formalism; for an overview of this method see [2]. One advantage this method has over the Dirac procedure is that it treats first- and second-class quantum constraints on an equal footing. The premise behind this method is that one first quantizes a classical system and then reduces the original Hilbert space via a suitable projection operator to a subspace in

*little@phys.ufl.edu

${ }^{\dagger}$ klauder@phys.ufl.edu

${ }^{\ddagger}$ Also Department of Mathematics 
which the constraints are satisfied to an appropriate level of accuracy; see 2 ] for a more complete discussion. ${ }^{1}$

Explicitly, we recall that the constraint algebra for classical gravity is given by

$$
\begin{aligned}
\left\{H_{a}(x), H_{b}(y)\right\} & =\delta_{, a}(x, y) H_{b}(x)-\delta_{, b}(x, y) H_{a}(x) \\
\left\{H_{a}(x), H(y)\right\} & =\delta_{, a}(x, y) H(x) \\
\{H(x), H(y)\} & =\delta_{, a}(x, y) g^{a b}(x) H_{b}(x)
\end{aligned}
$$

where $H$ is the Hamiltonian constraint and $H_{a}$ are the diffeomorphism constraints. While (11) and (2) maintain a first-class structure upon quantization, (31) morphs into a partially second-class constraint due to the metric factor; see [3] for a further discussion. It is this particular behavior of gravity that we mimic with a simple, few-degrees of freedom quantum mechanical system.

\section{Classical Formulation}

The initial problem under consideration is based on using the three components of angular momentum, $j_{i}, i \in\{1,2,3\}$, as constraints. As a modification of this problem, we multiply each component of the angular momentum by a suitable, non-vanishing variable. It is convenient to describe both of these examples in parallel.

\subsection{Basic Constraints}

We discuss a system of constraints that force the angular momentum $j_{i}, i \in$ $\{1,2,3\}$, to vanish. With the angular momentum $j_{i} \equiv \epsilon_{i j k} q^{j} p_{k}$ (summation implied), the action integral we choose is given by

$$
I_{1}=\int\left(p_{a} \dot{q}^{a}-\lambda^{b} j_{b}\right) d t
$$

where $\lambda^{b}$ denotes the Lagrange multipliers to enforce the constraints. Note that the Hamiltonian $H(p, q)$ is identically zero in this example. This form has been chosen for simplicity so we can focus directly on the issues surrounding the constraints.

\footnotetext{
${ }^{1}$ As a note of interest, one could consider the case of the simplest example of a classical second-class constraint, namely, $p=q=0$. When mapped into a corresponding quantum system, the constraints are satisfied by a projection operator onto the ground state of an harmonic oscillator with $\omega=1, \mathbb{E}\left(P^{2}+Q^{2} \leq \hbar\right)=|0\rangle\langle 0|$ [2].
} 
From the definition of the $j_{i}$ 's, one can immediately determine the Poisson algebra, given as usual by

$$
\left\{j_{i}, j_{j}\right\}=\epsilon_{i j k} j_{k}
$$

Since this bracket yields a Lie algebra, our system is clearly a closed first-class constraint system [4].

\subsection{Modified Constraints}

The action for our choice of the modified system is very similar in form, i.e.,

$$
I_{2}=\int\left(p_{a} \dot{q}^{a}-\lambda^{b} l_{b}\right) d t
$$

where the essential change resides in the definition of the variables $l_{i}$. For some smooth, non-vanishing function, $f$, we define (note: $q_{1} \equiv q^{1}$, etc.)

$$
l_{i} \equiv f\left(p_{1}, p_{2}, q_{1}, q_{2}\right) j_{i}
$$

for all $i$, and choose for further study the particular example for which

$$
f\left(p_{1}, p_{2}, q_{1}, q_{2}\right) \equiv \alpha+(\beta / \tilde{\hbar})\left(p_{1}^{2}+q_{1}^{2}\right)+(\gamma / \tilde{\hbar})\left(p_{2}^{2}+q_{2}^{2}\right) .
$$

The symbol $\tilde{\hbar}$ is a fixed constant equal in value to the physical value of Planck's constant $\hbar$, namely $1.06 \times 10^{-27}$ erg-sec. When the classical limit is called for, and thus Planck's constant $\hbar \rightarrow 0$, we emphasize that $\tilde{\hbar}$ retains its original numerical value. We have chosen such a small divisor to emphasize the quantum corrections; different divisors can be considered by rescaling $\beta$ and $\gamma$.

Since $\hbar$ dependence will play a role in our analysis, we do not set $\hbar=1$. In our choice of units, both $p$ and $q$ have the dimensions of $\sqrt{\hbar}$. Therefore, the division of $\beta$ and $\gamma$ by $\tilde{\hbar}$ has been chosen so that $\alpha, \beta$, and $\gamma$ can all be dimensionless. We further suppose that $\alpha+\beta+\gamma=1$ and $0<\alpha \leq 1$; hence, $0<\alpha \leq f$. Due to an evident $\beta \leftrightarrow \gamma$ symmetry it suffices hereafter to consider $0 \leq \gamma<\beta$. (The special case of $\gamma=\beta$ is considered later.)

We recognize, in this simple case, that we could absorb the factor $f$ by a redefinition of the Lagrange multipliers in (6). In more complicated systems (e.g., gravity) this simplification is either very difficult or perhaps impossible. Therefore, as a further analog, we retain $f$ as a part of $l_{a}$. A straightforward 
analysis leads to

$$
\begin{aligned}
\left\{l_{i}, l_{j}\right\}= & \left\{f j_{i}, f j_{j}\right\} \\
= & f^{2}\left\{j_{i}, j_{j}\right\}+f\left\{j_{i}, f\right\} j_{j}+f\left\{f, j_{j}\right\} j_{i}+\{f, f\} j_{i} j_{j} \\
= & f \epsilon_{i j k} l_{k}+\left\{j_{i}, f\right\} l_{j}+\left\{f, j_{j}\right\} l_{i} \\
= & f \epsilon_{i j k} l_{k}+\epsilon_{i a b}\left[-q^{a} \partial f / \partial q^{b}+p_{b} \partial f / \partial p^{a}\right] l_{j} \\
& -\epsilon_{j a b}\left[-q^{a} \partial f / \partial q^{b}+p_{b} \partial f / \partial p^{a}\right] l_{i} .
\end{aligned}
$$

Since $f>0$, our modified set of constraints is classified as open, first-class.

For the particular example of interest,

$$
\begin{aligned}
\left\{l_{i}, l_{j}\right\}= & {\left[\alpha+(\beta / \tilde{\hbar})\left(p_{1}^{2}+q_{1}^{2}\right)+(\gamma / \tilde{\hbar})\left(p_{2}^{2}+q_{2}^{2}\right)\right] \epsilon_{i j k} l_{k} } \\
& -2(\beta / \tilde{\hbar}) \epsilon_{i a 1}\left(q^{a} q_{1}+p_{a} p_{1}\right) l_{j}+2(\beta / \tilde{\hbar}) \epsilon_{j a 1}\left(q^{a} q_{1}+p^{a} p_{1}\right) l_{i} \\
& -2(\gamma / \tilde{\hbar}) \epsilon_{i a 2}\left(q^{a} q_{2}+p_{a} p_{2}\right) l_{j}+2(\gamma / \tilde{\hbar}) \epsilon_{j a 2}\left(q^{a} q_{2}+p_{a} p_{2}\right) l_{i}
\end{aligned}
$$

which is manifestly an open first-class constraint algebra when $\beta>0$. The equivalence of the two models is evident since $j_{k}=0$ implies that $l_{k}=0$, for all $k$, and vice versa. Observe, as $\beta \rightarrow 0$, and thus $\alpha \rightarrow 1$, the $l$-constraints pass smoothly to the $j$-constraints.

\section{Quantization}

We now proceed by canonically quantizing our model; a path integral quantization is discussed in Section 4. Initially, let us assume our chosen canonical coordinates are Cartesian coordinates suitable for quantization [5], and thus we promote the variables $\left(p_{i}, q^{j}\right)$ to a set of irreducible, self-adjoint operators $\left(P_{i}, Q^{j}\right)$ that obey the standard Heisenberg commutation relation,

$$
\left[Q^{i}, P_{j}\right]=i \hbar \delta_{j}^{i}
$$

Following the standard Dirac procedure of constraint quantization, our constraints are chosen as suitable self-adjoint functions of the basic operators, namely

$$
\begin{aligned}
& j_{i} \mapsto J_{i}=\epsilon_{i j k} Q_{j} P_{k} \\
& l_{i} \mapsto L_{i}=\alpha J_{i}+(\beta / 2 \hbar)\left[\left(P_{1}^{2}+Q_{1}^{2}\right) J_{i}+J_{i}\left(P_{1}^{2}+Q_{1}^{2}\right)\right] \\
& +\quad(\gamma / 2 \hbar)\left[\left(P_{2}^{2}+Q_{2}^{2}\right) J_{i}+J_{i}\left(P_{2}^{2}+Q_{2}^{2}\right)\right],
\end{aligned}
$$

where $\alpha+\beta+\gamma=1$. In dealing with the quantum theory, we drop the distinction between $\tilde{\hbar}$ and $\hbar$. A simple calculation shows that any other choice of factor-ordering to define $L_{i}$ is equivalent to the chosen form. In particular, normal ordering of the expressions is equivalent to a redefinition of the parameters $\alpha, \beta$, and $\gamma$. 


\subsection{Quantum Classification}

As in the classical case we can determine the classification of the $J_{i}$ 's based on their commutator structure as given by

$$
\left[J_{i}, J_{j}\right]=i \hbar \epsilon_{i j k} J_{k}
$$

This identity, which follows the classical Poisson bracket, implies that the $J_{i}$ 's form a quantum system of closed, first-class constraints.

On the other hand, if we look at the commutators for the $L_{i}$ 's, as shown below, we find a different outcome altogether. In what follows, we have introduced

$$
A \equiv(\alpha / 2)+(\beta / 2 \hbar)\left(P_{1}^{2}+Q_{1}^{2}\right)+(\gamma / 2 \hbar)\left(P_{2}^{2}+Q_{2}^{2}\right)
$$

which means that

$$
L_{j} \equiv A J_{j}+J_{j} A
$$

Observe that the operator $A>0$. However, unlike the case of the classical constraints, the equation $J_{l}|\phi\rangle=0$, for some $l$, does not generally imply that $L_{l}|\phi\rangle=0$, whenever $\beta>0$. The commutator of $L_{i}$ and $L_{j}$ reads

$$
\begin{gathered}
{\left[L_{i}, L_{j}\right]=i \hbar \epsilon_{i j k} A J_{k} A+\left(J_{i} A J_{j}-J_{j} A J_{i}\right) A+A\left(J_{i} A J_{j}-J_{j} A J_{i}\right)} \\
+\left(J_{i} A^{2} J_{j}-J_{j} A^{2} J_{i}\right) .
\end{gathered}
$$

Unlike the $J_{i}$ 's, the $L_{i}$ 's are no longer first-class constraints, but are partially second class, a consequence of the open first-class nature of their classical system. The second-class nature of the $L_{i}$ 's is exhibited below.

\subsection{Restricted Quantum Problem}

A complete analysis of the $J$ - and $L$-algebras is sufficiently complicated that we seek a simplification. To this end, let us introduce conventional annihilation and creation operators given by

$$
\begin{aligned}
a_{j} & =\left(Q_{j}+i P_{j}\right) / \sqrt{2 \hbar}, \\
a_{j}^{\dagger} & =\left(Q_{j}-i P_{j}\right) / \sqrt{2 \hbar} .
\end{aligned}
$$

If we define

$$
N=a_{1}^{\dagger} a_{1}+a_{2}^{\dagger} a_{2}+a_{3}^{\dagger} a_{3}
$$


as the total number operator, it is evident that

$$
\left[J_{j}, N\right]=0, \quad\left[L_{j}, N\right]=0,
$$

for all $j$, and thus both sets $\left\{J_{i}\right\}$ and $\left\{L_{i}\right\}$ are number conserving. This conservation implies that we can study the fulfillment of both sets of constraints in each of the number-operator subspaces independently of one another. We observe that the subspace for which $N=0$ consists of just a single state, and this state is an eigenvector of each $J_{i}$ as well as each $L_{i}, i \in\{1,2,3\}$, all with eigenvalue zero. In the interest of simplicity in this paper, we restrict our attention to the lowest nontrivial subspace in which the constraints $J_{i}=0$ are satisfied on a non-vanishing subspace. In particular, we confine our attention to a subspace of the entire Hilbert space corresponding to an eigenvalue of the total number operator of two. Note that the subspace of interest is six-dimensional and is spanned by the six vectors given by the two representatives

$$
\begin{aligned}
& |1,1,0\rangle=a_{1}^{\dagger} a_{2}^{\dagger}|0\rangle, \quad \text { etc., } \\
& |2,0,0\rangle=(1 / \sqrt{2})\left(a_{1}^{\dagger}\right)^{2}|0\rangle, \quad \text { etc. },
\end{aligned}
$$

where as usual $|0\rangle(=|0,0,0\rangle)$ denotes the no particle state for which $a_{j}|0\rangle=$ 0 for all $j$.

\subsection{Construction of the Projection Operators}

Having chosen a particular subspace of the Hilbert space on which to focus our attention, we now have the information needed to construct the projection operators. We do so by first determining all possible eigenvectors and eigenvalues of the equation

$$
\Sigma_{i=1}^{3} \Phi_{i}^{2}|\psi\rangle=\nu|\psi\rangle
$$

where $\Phi_{i}=J_{i}$ or $L_{i}$, and $\nu \geq 0$. We also consider the equation

$$
\Phi_{3}|\psi\rangle=\eta|\psi\rangle
$$

where $\Phi_{3}=J_{3}$ or $L_{3}$, and $\eta$ is real.

\section{4 $J_{i}$ Considerations}

We begin with our quantum first class system. The well-known eigenvectors and eigenvalues for the $N=2$ subspace are presented in Table 1 (modulo normalization factors for the eigenstates). 
Table 1: $J_{3}$ and $J^{2}$ Eigenstates and Eigenvalues

\begin{tabular}{|c|c|c|}
\hline Eigenstates & $J_{3}$ Eigenvalues & $J^{2}$ Eigenvalues \\
\hline \hline$|1,1,0\rangle-\frac{i}{\sqrt{2}}|2,0,0\rangle+\frac{i}{\sqrt{2}}|0,2,0\rangle$ & $2 \hbar$ & $6 \hbar^{2}$ \\
\hline$|1,0,1\rangle+i|0,1,1\rangle$ & $\hbar$ & $6 \hbar^{2}$ \\
\hline$|2,0,0\rangle+|0,2,0\rangle-2|0,0,2\rangle$ & 0 & $6 \hbar^{2}$ \\
\hline$|1,0,1\rangle-i|0,1,1\rangle$ & $-\hbar$ & $6 \hbar^{2}$ \\
\hline$|1,1,0\rangle+\frac{i}{\sqrt{2}}|2,0,0\rangle-\frac{i}{\sqrt{2}}|0,2,0\rangle$ & $-2 \hbar$ & $6 \hbar^{2}$ \\
\hline \hline$|2,0,0\rangle+|0,2,0\rangle+|0,0,2\rangle$ & 0 & 0 \\
\hline
\end{tabular}

As expected, the operators $J_{3}$ and $J^{2} \equiv \Sigma J_{i}^{2}$ share a common basis, since they can be simultaneously diagonalized. Based on this information, we choose our projection operator as

$$
\begin{aligned}
\mathbb{E}_{J} & \equiv \mathbb{E}\left(\Sigma_{i=1}^{3} J_{i}^{2} \leq \hbar^{2}\right) \\
& =\frac{1}{3}(|2,0,0\rangle+|0,2,0\rangle+|0,0,2\rangle)(\langle 2,0,0|+\langle 0,2,0|+\langle 0,0,2|) \\
& \equiv\left|O_{J}\right\rangle\left\langle O_{J}\right| .
\end{aligned}
$$

Note that the factor $\hbar^{2}$ in the argument of the projection operator has been selected to exclude any integer spin value other than zero; clearly, other values for that parameter (less than $6 \hbar^{2}$ ) would do equally well.

We can now see how the projection operator determines the one-dimensional physical Hilbert space $\mathcal{H}_{P}$ within the six-dimensional original Hilbert space (confining attention to the two-particle subspace) as follows. Consider a general vector $|\psi\rangle_{2} \in \mathcal{H}_{2}$ (the two-particle subspace). Then

$$
\begin{aligned}
\mathbb{E}_{J}|\psi\rangle_{2} & =\left|O_{J}\right\rangle\left\langle O_{J} \mid \psi\right\rangle_{2}=|\psi\rangle_{2 P} \in \mathcal{H}_{2 P}, \\
J_{i} \mathbb{E}_{J}|\psi\rangle_{2} & =J_{i}\left|O_{J}\right\rangle\left\langle O_{J} \mid \psi\right\rangle_{2}=0 \in \mathcal{H}_{2 P}, \quad i=1,2,3 .
\end{aligned}
$$

This result implies that our constraint condition $\left(J_{j}|\psi\rangle_{2 P}=0\right.$, for all $j$ ) is satisfied by every vector in the physical Hilbert space. Consequently, we have successfully quantized our closed first-class constraints, in this restricted Hilbert space. It is apparent for this example that a similar story would apply in all even-particle subspaces of the original Hilbert space.

\section{5 $\quad L_{i}$ Considerations}

We next follow a similar procedure for the $L_{i}$ operators. To further simplify matters, we choose $\gamma \equiv \beta / 2$. The eigenvectors and eigenvalues of $L_{3}$ and $L^{2} \equiv \Sigma L_{i}^{2}$ are presented in Tables 2 and 3 (modulo normalization factors for the eigenstates). 
Table 2: $L_{3}$ Eigenstates and Eigenvalues

\begin{tabular}{|c||c|}
\hline$L_{3}$ Eigenstates & $L_{3}$ Eigenvalues \\
\hline \hline$|1,1,0\rangle-\imath a|2,0,0\rangle+\imath a^{\prime}|0,2,0\rangle$ & $\hbar \sqrt{4+24 \beta+37 \beta^{2}}$ \\
\hline$|1,0,1\rangle+\imath|0,1,1\rangle$ & $\hbar(1+3 / 2 \beta)$ \\
\hline$\frac{2+5 \beta}{2+7 \beta}|2,0,0\rangle+|0,2,0\rangle-\frac{2\left(4+24 \beta+37 \beta^{2}\right)}{(2+7 \beta)^{2}}|0,0,2\rangle$ & 0 \\
\hline$|1,0,1\rangle-\imath|0,1,1\rangle$ & $-\hbar(1+3 / 2 \beta)$ \\
\hline$|1,1,0\rangle+\imath a|2,0,0\rangle-\imath a^{\prime}|0,2,0\rangle$ & $-\hbar \sqrt{4+24 \beta+37 \beta^{2}}$ \\
\hline \hline$\frac{2+5 \beta}{2+7 \beta}|2,0,0\rangle+|0,2,0\rangle+|0,0,2\rangle$ & 0 \\
\hline
\end{tabular}

Here $a$ and $a^{\prime}$ are defined as,

$$
\begin{aligned}
a & =\frac{2+7 \beta}{\sqrt{8+48 \beta+74 \beta^{2}}} \\
a^{\prime} & =\frac{2+5 \beta}{\sqrt{8+48 \beta+74 \beta^{2}}} .
\end{aligned}
$$

Observe how these $L_{3}$ eigenstates and eigenvalues all pass smoothly to the appropriate $J_{3}$ eigenstates and eigenvalues as $\beta \rightarrow 0$.

We now turn our attention to the eigenvectors and eigenvalues of $L^{2}$, and in this discussion we offer exact and approximate statements. The first three entries in Table 3, below, associated with the states $|1,1,0\rangle$, etc., are exact in both the eigenvectors and eigenvalues. The product of the last three eigenvalues, associated with the three states $|2,0,0\rangle$, etc., is given exactly by the expression $288 \beta^{2} \gamma^{2}(\beta-\gamma)^{2}$. This relation implies that (at least) one eigenvalue is zero if $\beta=0$, or $\gamma=0$, or $\beta=\gamma$. Thus we confine attention to the range $0 \leq \gamma<\beta$, and focus on the case where $\gamma=\beta / 2$. Unfortunately, the exact forms of the last three eigenvectors and eigenvalues are exceedingly complicated functions of $\beta$ and provide little insight into their small $\beta$ behavior. To present this information, we use their Taylor series to first non-zero order in $\beta$. The exact nature of these three eigenvalues can be observed in Figs. 1 and 2.

Table 3: $L^{2}$ Eigenstates and Eigenvalues 


\begin{tabular}{|c|c|}
\hline$\Sigma L_{i}^{2}$ Eigenstates & $\Sigma L_{i}^{2}$ Eigenvalues \\
\hline$\overline{|0,1,1\rangle}$ & $\overline{\hbar^{2}\left(6+15 \beta+45 \beta^{2} / 4\right)}$ \\
\hline$|1,0,1\rangle$ & $\hbar^{2}\left(6+24 \beta+57 \beta^{2} / 2\right)$ \\
\hline$|1,1,0\rangle$ & $\hbar^{2}\left(6+33 \beta+189 \beta^{2} / 4\right)$ \\
\hline \begin{tabular}{c|}
$b|2,0,0\rangle+b^{\prime}|0,2,0\rangle+|0,0,2\rangle$ \\
\end{tabular} & $\hbar^{2}\left(6+6(4+\sqrt{3}) \beta+O\left(\beta^{2}\right)\right)$ \\
\hline \begin{tabular}{c|}
$c|2,0,0\rangle+c^{\prime}|0,2,0\rangle+|0,0,2\rangle$ \\
\end{tabular} & $\hbar^{2}\left(6+6(4-\sqrt{3}) \beta+O\left(\beta^{2}\right)\right)$ \\
\hline 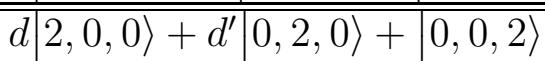 & $\overline{\hbar^{2}\left(\beta^{6} / 2+O\left(\beta^{7}\right)\right)}$ \\
\hline
\end{tabular}

Here $b, b^{\prime}, c, c^{\prime}, d$ and $d^{\prime}$ are approximated by

$$
\begin{aligned}
b & =(-2-\sqrt{3})-\frac{7}{4}(2+\sqrt{3}) \beta+O\left(\beta^{2}\right) \\
b^{\prime} & =(1+\sqrt{3})+\left(\frac{1}{2}+\frac{3 \sqrt{3}}{4}\right) \beta+O\left(\beta^{2}\right) \\
c & =(-2+\sqrt{3})-\frac{7}{4}(-2+\sqrt{3}) \beta+O\left(\beta^{2}\right), \\
c^{\prime} & =(1-\sqrt{3})+\left(\frac{1}{2}-\frac{3 \sqrt{3}}{4}\right) \beta+O\left(\beta^{2}\right) \\
d & =1-2 \beta+O\left(\beta^{2}\right) \\
d^{\prime} & =1-\beta+O\left(\beta^{2}\right) .
\end{aligned}
$$

Clearly, as $\beta \rightarrow 0$, and thus $\alpha \rightarrow 1$, we recover all the properties of $J_{3}$ and $\Sigma J_{i}^{2}$.

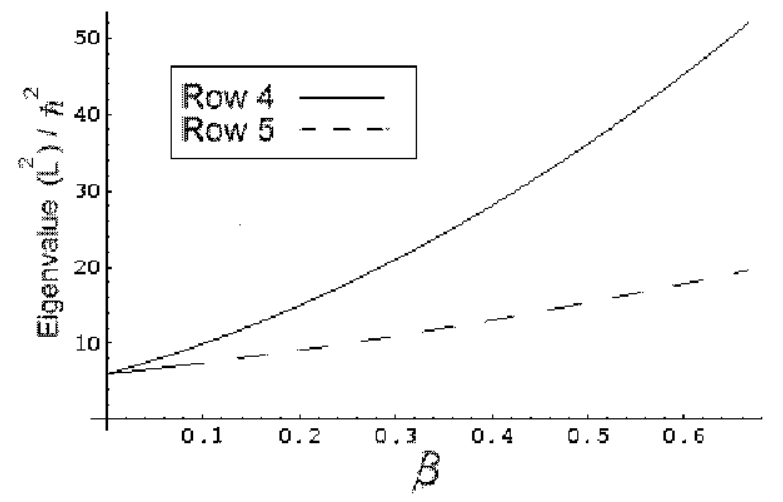

Fig.1 Exact $L^{2}$ Eigenvalues of Row 4 and Row 5, Table 3, as functions of $\beta$ 


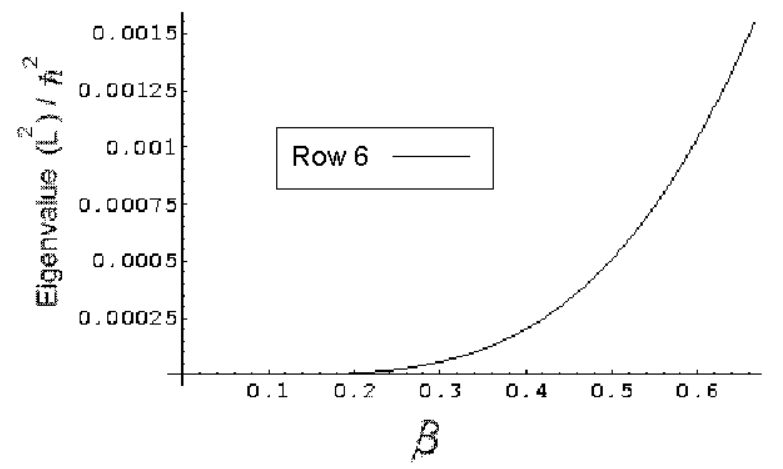

Fig.2 Exact $L^{2}$ Eigenvalue of Row 6, Table 3, as a function of $\beta$

Unlike $J_{3}$ and $\Sigma J_{i}^{2}$, the operators $L_{3}$ and $\Sigma L_{i}^{2}$ do not share a common basis. An important feature of the $L^{2}$ operator is the absence of zero in its spectrum when $\beta>0$. This is a clear reflection of the partial second-class nature of the $L_{i}$ constraints. However, the spectrum of $\Sigma L_{i}^{2}$ admits a lowest possible eigenvalue, and from that state (or states) we construct our projection operator. To ensure that $\alpha>0$, when $\gamma=\beta / 2$, it is necessary to restrict $\beta$ so that $0 \leq \beta<2 / 3$. It follows that the lowest eigenvalue of $\Sigma L_{i}^{2}$ is non-degenerate and is represented by the last entry in Table 3 (row 6). Thus, in this case, we are led to adopt the projection operator given by

$$
\begin{aligned}
\mathbb{E}_{L} & \equiv \mathbb{E}\left(\sum_{i=1}^{3} L_{i}^{2} \leq \delta(\hbar)^{2}\right) \\
& =|M|^{2}\left(d|2,0,0\rangle+d^{\prime}|0,2,0\rangle+|0,0,2\rangle\right)\left(d\langle 2,0,0|+d^{\prime}\langle 0,2,0|+\langle 0,0,2|\right) \\
& \equiv\left|O_{L}\right\rangle\left\langle O_{L}\right|,
\end{aligned}
$$

where $M=1 / \sqrt{d^{2}+d^{2}+1}$ is a normalization factor. In this expression we may set

$$
\delta(\hbar)^{2}=\beta \hbar^{2}
$$

which lies between the lowest and next to lowest eigenvalues of $\Sigma L_{i}^{2}$ when $0 \leq \beta<2 / 3$ (i.e.. Eigenvalue $\left(L^{2}\right)$ row $\left.6 \leq \delta(\hbar)^{2}\right)$. Other choices for $\delta(\hbar)^{2}$ are possible as long as the resultant $\mathbb{E}_{L}$ is the same. Again by taking an arbitrary vector $|\psi\rangle_{2} \in \mathcal{H}_{2}$, we can project that vector into the two-dimensional physical Hilbert space by acting with $\mathbb{E}_{L}$ on the vector. In symbols, we have

$$
\mathbb{E}_{L}|\psi\rangle_{2} \equiv|\psi\rangle_{2 P}=\left|O_{L}\right\rangle\left\langle O_{L} \mid \psi\right\rangle_{2} \in \mathcal{H}_{2 P}
$$

Unlike our first class constraint system, the constraint equation $L_{i}|\psi\rangle_{2 P} \neq$ 0 for all $i \in\{1,2,3\}$, unless $|\psi\rangle_{2 P}=0$. However, this behavior is a general characteristic of quantum second-class constraints. 
With the foregoing discussion, we have successfully dealt with a quantum second-class constraint in the context of our restricted Hilbert space. An analogous discussion would take place in higher-order, even-particle subspaces.

\section{Path Integral Approach}

In Section 3, we discussed the canonical quantization of the model, however, this is not the only procedure to quantize the system. We now focus our attention on a path integral approach. Before discussing the projection operator in this language, let us first show how the Faddeev method [6] would treat this example. The formal starting relation

$$
\int e^{(i / \hbar) \int\left[p_{a} \dot{q}^{a}-\lambda^{b} f j_{b}\right] d t} \mathcal{D} p \mathcal{D} q \mathcal{D} \lambda
$$

is replaced with the gauge-fixed expression

$$
\int e^{(i / \hbar) \int p_{a} \dot{q}^{a} d t} \Pi_{b} \Pi_{c} \delta\left\{\chi^{b}\right\} \delta\left\{f j_{b}\right\} \operatorname{det}\left\{\chi^{b}, f j_{c}\right\} \mathcal{D} p \mathcal{D} q
$$

where $\chi^{b}(p, q)$ is some appropriate gauge choice. A simple identity leads to

$$
\int e^{(i / \hbar) \int p_{a} \dot{q}^{a} d t} \Pi_{b} \Pi_{c} \delta\left\{\chi^{b}\right\} \frac{\delta\left\{j_{b}\right\}}{\Pi_{t} f^{3}} \operatorname{det}\left(\left\{\chi^{b}, f\right\} j_{c}+\left\{\chi^{b}, j_{c}\right\} f\right) \mathcal{D} p \mathcal{D} q .
$$

The first term in the determinant is zero by the $\delta$ functional of the $j$ 's. The second term is a $3 \times 3$ matrix multiplied by a scalar $f$, and therefore becomes.

$$
\int e^{(i / \hbar) \int p_{a} \dot{q}^{a} d t} \Pi_{b} \Pi_{c} \delta\left\{\chi^{b}\right\} \frac{\delta\left\{j_{b}\right\}}{\Pi_{t} f^{3}} \Pi_{t} f^{3} \operatorname{det}\left\{\chi^{b}, j_{c}\right\} \mathcal{D} p \mathcal{D} q
$$

We observe that all the factors of $f$ completely cancel. As one can see the Faddeev method is insensitive to the definition of $f$, only that it be non-zero. Hence, this method considers the $j$ 's and l's as identical constraints. This is not surprising since using this method we are satisfying the constraints classically, and as shown in Section 2, the constraints behave the same in the classical regime.

It is often stated that the results of (27) are correct up to terms of order $\hbar$. While this may be true, the goal of quantization is to obtain correct $\hbar$ dependence. Otherwise Bohr-Sommerfeld [7] would be an adequate quantization procedure. Based on the discussion of Section 3 we can acquire the correct $\hbar$ dependence in the present case. 
A coherent state path integral can also be used to calculate the matrix elements of the projector as shown in previous works [2]. Let us begin with a preliminary equation, namely,

$$
\begin{aligned}
\left\langle\vec{z}^{\prime \prime}\left|T e^{-(i / \hbar) \int \lambda^{a} J_{a} d t}\right| \vec{z}^{\prime}\right\rangle & \\
& =\mathcal{M} \int \exp \left\{(i / \hbar) \int\left(\left(p_{a} \dot{q}^{a}-q^{a} \dot{p}_{a}\right) / 2-\lambda^{a} j_{a}\right) d t\right\} \mathcal{D} p \mathcal{D} q \\
& =N^{\prime \prime} N^{\prime} \exp \left\{\vec{z}^{\prime \prime *} \cdot e^{-(i / \hbar) \vec{\theta} \cdot \vec{j}} \vec{z}^{\prime}\right\}
\end{aligned}
$$

where $\mathcal{M}, N^{\prime \prime}$, and $N^{\prime}$ are normalization factors, $\vec{z} \equiv(\vec{q}+i \vec{p}) / \sqrt{2 \hbar}, \vec{j}$ is a $3 \times 3$ matrix representation of the rotation algebra, $T$ denotes time ordering, and $\vec{\theta}$ is a suitable functional of $\left\{\lambda^{a}(\cdot)\right\}$.

Following [2], we could integrate over $\vec{\lambda}$ with respect to a suitable measure $R(\vec{\lambda})$ to create the desired projection operator. However, it is equivalent and simpler to proceed as follows,

$$
\begin{aligned}
\mathcal{K}_{J}\left(\vec{z}^{\prime \prime} ; \vec{z}^{\prime}\right) & \equiv\left\langle\vec{z}^{\prime \prime}\left|\mathbb{E}\left(J^{2} \leq \hbar^{2}\right)\right| \vec{z}^{\prime}\right\rangle \\
& =\int\left\langle\vec{z}^{\prime \prime}\left|e^{-(i / \hbar) \vec{\theta} \cdot \vec{J}}\right| \vec{z}^{\prime}\right\rangle d \mu(\theta),
\end{aligned}
$$

where $d \mu(\theta)$ is the normalized Haar measure of $S O(3)$. Consequently,

$$
\begin{aligned}
\mathcal{K}_{J}\left(\vec{z}^{\prime \prime} ; \vec{z}^{\prime}\right) & =\left(N^{\prime \prime} N^{\prime} / 2\right) \int \exp \left\{\sqrt{\vec{z}^{\prime \prime * 2}} \sqrt{\vec{z}^{\prime 2}} \cos \theta\right\} d \cos \theta \\
& =N^{\prime \prime} N^{\prime} \frac{\sinh \sqrt{\vec{z}^{\prime \prime * 2} \vec{z}^{\prime 2}}}{\sqrt{\vec{z}^{\prime \prime * 2} \vec{z}^{\prime 2}}} \\
& =N^{\prime \prime} N^{\prime}\left[1+\frac{\vec{z}^{\prime \prime * 2} \vec{z}^{\prime 2}}{3 !}+\frac{\left(\vec{z}^{\prime \prime * 2} \vec{z}^{\prime 2}\right)^{2}}{5 !}+\ldots\right] \\
& =\left\langle\vec{z}^{\prime \prime} \mid 0\right\rangle\left\langle 0 \mid \vec{z}^{\prime \prime}\right\rangle+\left\langle\vec{z}^{\prime \prime} \mid O_{J}\right\rangle\left\langle O_{J} \mid \vec{z}^{\prime}\right\rangle+\ldots
\end{aligned}
$$

From (31) we can deduce that the physical Hilbert space for every even particle sector is one-dimensional, while in the odd particle sectors the physical Hilbert space is empty, as conjectured in Section 3.

We can also construct the fundamental kernel for the modified case using the results of Section 3. Specifically,.

$$
\begin{aligned}
\mathcal{K}_{L}\left(\vec{z}^{\prime \prime} ; \vec{z}^{\prime}\right) & =\left\langle\vec{z}^{\prime \prime} \mid 0\right\rangle\left\langle 0 \mid \vec{z}^{\prime}\right\rangle+\left\langle\vec{z}^{\prime \prime} \mid O_{L}\right\rangle\left\langle O_{L} \mid \vec{z}^{\prime}\right\rangle+\ldots \\
& =N^{\prime \prime} N^{\prime}\left[1+\frac{\left(d z_{1}^{\prime \prime * 2}+d^{\prime} z_{2}^{\prime \prime * 2}+z_{3}^{\prime \prime * 2}\right)\left(d z_{1}^{\prime 2}+d^{\prime} z_{2}^{\prime 2}+z_{3}^{\prime 2}\right)}{2 !\left(d^{2}+d^{\prime 2}+1\right)}+\ldots\right] .
\end{aligned}
$$


Although the full nature of this kernel is unknown at present, we expect that every higher even sector contained in $\mathcal{K}_{L}$ will also yield a one-dimensional physical Hilbert space, based on the observed nature of the $J$ 's.

\section{Summary}

Motivated by the quantization of gravity, we have considered a system that has classically first-class constraints; however, upon quantization it transforms partially to second class. In the two-particle subspace and for all $\beta>0$, our modified system has the characteristic feature that for a suitably chosen $\delta(\hbar)^{2}$, which generally depends on $\beta$, we have

$$
0<{ }_{2 P}\left\langle\psi\left|\Sigma_{i} L_{i}^{2}\right| \psi\right\rangle_{2 P} \leq \delta(\hbar)^{2}{ }_{2 P}\langle\psi \mid \psi\rangle_{2 P},
$$

an attribute shared by all quantum second-class constraints. If we were to take the limit $\delta(\hbar) \rightarrow 0$, our projection operator would vanish, resulting in an empty physical Hilbert space. Thus, as part of the general theory of the projection operator method [2], we do not take such a limit. Nevertheless, we observe that all properties of the $L$-constraints pass smoothly to those of the $J$-constraints as $\beta \rightarrow 0$, including the dimensionality of the physical Hilbert space.

The results we have obtained could be extended to all particle sectors that admit a lowest possible eigenvalue. One may also be interested in extending our procedure to models with additional degrees of freedom, and finally extending the arguments to include fields.

\section{Acknowledgments}

Wayne Bomstad is thanked for his comments and encouragement on this paper, and the suggestions of Jan Govaerts and Sergei Shabanov proved helpful. JSL would also like to acknowledge the University of Florida's Alumni Fellowship Program for support.

\section{References}

[1] P.A.M. Dirac, Lectures on Quantum Mechanics, (Belfer Graduate School of Science, Yeshiva University, New York, 1964).

[2] J.R. Klauder, "Quantization of Constrained Systems", Lect. Notes Phys. 572, 143-182 (2001); "Coherent State Quantization of Constraint Systems", Ann. Phys. 254 419-453 (1997). 
[3] J. Anderson, in Proceedings of the First Eastern Theoretical Physics Conference, University of Virginia 1962, edited by M.E. Rose (Gordan and Breach, New York, 1963), p.387.

[4] D.M. Gitman and I.V. Tyutin, Quantization of Fields with Constraints (Springer-Verlag Berlin Heidelberg 1990); J. Govaerts, Hamiltonian Quantisation and Constrained Dynamics (Leuven University Press, Belgium, 1991); M. Henneaux and C. Teitelboim, Quantization of Gauge Systems, (Princeton University Press, NJ, 1992).

[5] P.A.M Dirac, The Principles of Quantum Mechanics, (Oxford Science Publications, 1999), 4th Edition, p. 114.

[6] L.D. Faddeev, "Feynman Integral for Singular Lagrangians", Theor. Math. Phys. 1, 1-13 (1969).

[7] R. Shankar, Principles of Quantum Mechanics, (Plenum Press, NY, 1994), 2nd Edition, p. 448. 